\title{
LA BIOECONOMÍA Y EL USO DE SISTEMAS DE INFORMACIÓN GEOGRÁFICA COMO ALTERNATIVA POLÍTICA, ÉTICA Y ECOLÓGICA PARA RESOLVER PROBLEMÁTICAS MEDIOAMBIENTALES
}

\section{Bioeconomics and the use of Geographic Information Systems as a political, ethical and ecological alternative to solve environmental problems}

Revista Trama

Volumen 9, número 2

Julio - Diciembre 2020

Página 206-225

ISSN: 1659-343X

https://revistas.tec.ac.cr/trama

Cristina Estrada Porras ${ }^{1}$

Fecha de recepción: 19 de abril, 2020

Fecha de aprobación: 3 de diciembre, 2020

Estrada, C. (2020). La bioeconomía y el uso de sistemas de información geográfica como alternativa política, ética y ecológica para resolver problemáticas medioambientales. Trama, Revista de ciencias sociales y humanidades, Volumen 9, (2), Julio-Diciembre, págs. 206-225.

DOI: https://doi.org/10.18845/tramarcsh.v9i2.5572

1. Estudiante de Ingeniería Forestal. Instituto Tecnológico de Costa Rica. Cartago, Costa Rica. 


\section{RESUMEN}

El capitalismo, sistema económico que actualmente es el orden social y económico de muchos países y una de las principales fuentes de contaminación global, añadido a la falta de conciencia ambiental de los gobiernos y la población, son algunas de las causas de la problemática medioambiental que atentan contra la vida de los seres vivos en el planeta. Conocer estas problemáticas y buscar alternativas para solventar este daño hacia la naturaleza son acciones importantes para practicar como humanidad. En este estudio se investigó sobre las problemáticas ambientales, conceptos filosóficos y alternativas políticas, éticas y ecológicas a partir de la consulta literaria y se seleccionaron casos de artículos y trabajos académicos que utilizaran Sistemas de Información Geográfica (SIG) para profundizar en la utilidad de dicha herramienta. Los resultados de dicha búsqueda evidencian que la bioeconomía es un sistema económico basado en la aplicación de conocimientos y tecnologías para la producción de bienes y servicios a partir del aprovechamiento sostenible de los recursos biológicos naturales; asimismo, demuestran mediante casos que los SIG, como instrumento tecnológico, son una gran herramienta para la elaboración de estudios ambientales y para la toma de decisiones en pro del ambiente. Por lo tanto, la bioeconomía y el uso de SIG son excelentes alternativas políticas, éticas y ecológicas para resolver problemáticas medioambientales.

Palabras clave: daño ambiental; medio ambiente; filosofía; economía; tecnología.

\section{ABSTRACT}

Capitalism, an economic system that is currently the social and economic order of many countries and one of the main sources of global pollution, added to the lack of environmental awareness of governments and the population, are some of the causes of the environmental problems that threaten the life of living beings on the planet. Knowing these problems and looking for alternatives to solve this damage to nature are important actions to practice as humanity. In this study, environmental problems, philosophical concepts and political, ethical and ecological alternatives were investigated from the literary consultation and cases of articles and academic works that used Geographic Information Systems (GIS) were selected to deepen the usefulness of said tool. The results of this search show that the bioeconomy is an economic system based on the application of knowledge and technologies for the production of goods and services from the sustainable use of natural biological resources; likewise, they demonstrate through cases that GIS, as a technological instrument, are a great tool for preparing environmental studies and for making decisions in favor of the environment. Therefore, the bioeconomy and the use of GIS are excellent political, ethical and ecological alternatives to solve environmental problems.

Keywords: environmental damage; environment; philosophy; economics; technology. 


\section{INTRODUCCIÓN}

La problemática ambiental es atribuida completamente al ser humano, por su egocentrismo y su insaciable deseo de consumo. Las personas han adquirido esta conducta debido al capitalismo que las lleva al consumismo. Este sistema económico es el causante de grandes cantidades de contaminantes, tanto sólidos, como líquidos y gaseosos, que originan la destrucción de la naturaleza, el agotamiento de los recursos naturales, la extinción de especies de flora y fauna, y que comunidades vulnerables enteras sean explotadas o destruidas para su beneficio, donde siempre gana la persona rica y la persona pobre es la mayor afectada ambientalmente.

Por ende, es importante implementar las prácticas políticas, éticas y ecológicas, donde el humano no se vea como un ego, sino como un eco, es decir, que considere que las demás especies compañeras, animales y vegetales, posean el mismo valor moral y sean respetadas. Esta ética ecológica tiene que ir de la mano con una política ecológica, la cual tendrá una economía política del ambiente, donde se elimina la desigualdad en la distribución de la riqueza y el poder, y se combate la degradación ambiental, para así, tener un desarrollo sostenible y socialmente justo.

La bioeconomía y los Sistemas de Información Geográfica (SIG) son una alternativa política, ética y ecológica que ayudarán al mejoramiento del planeta. La bioeconomía por su parte promueve el aprovechamiento y uso inteligente de recursos naturales con la utilización de conocimientos y tecnologías para una producción sostenible de bienes y servicios; y los SIG son esas tecnologías que ayudarán a la sostenibilidad, ya que por medio de estos sistemas informáticos se pueden realizar diferentes estudios/ mapeos a nivel nacional e internacional sobre la condición ambiental de ciertas zonas y así tomar decisiones para el mejoramiento ambiental.

Por lo tanto, este artículo pretende conocer el daño ambiental que está afectando al planeta y, así, proponer alternativas políticas, éticas y ecológicas, como lo son la bioeconomía y los SIG, para resolver esta problemática medioambiental.

Por lo tanto, este artículo pretende describir daños ambientales que están afectando al planeta y sus causas, así como también, conocer alternativas políticas, éticas y ecológicas, tales como, la bioeconomía en conjunto con el uso de tecnologías (SIG), para resolver problemáticas medioambientales. Además, analizar los SIG a profundidad por medio de un estudio de casos, los cuales utilizaron esta herramienta para realizar sus investigaciones y mencionar sus principales beneficios en los estudios ambientales. 


\section{MARCO TEÓRICO}

\section{PROBLEMÁTICA MEDIOAMBIENTAL}

Cuervo Bernal y Gutiérrez Rodríguez (2016) citan que

"el planeta Tierra vive un período de intensas transformaciones técnico-científicas como contrapartida de las cuales se han engendrado fenómenos de desequilibrio ecológico que amenazan, a corto plazo, si no se le pone remedio, la vida sobre su superficie".

Esto debido a la contaminación ambiental tan grave que los humanos han causado al planeta, por no tener una relación humanonaturaleza ecológica, si no egocéntrica, donde los humanos ven a la naturaleza como un objeto o una herramienta la cual está completamente para servirlos como persona dueña del planeta.

Igualmente, mencionan que el consumismo y capitalismo son la verdadera causa, las cuales originan la destrucción del medio ambiente, el agotamiento de los recursos naturales y una extinción masiva de especies, además de las comunidades que son destruidas y/o explotadas y que los beneficios que se generan no se dividen equitativamente, provocando una brecha gigante entre las personas ricas y las personas pobres, esta es una realidad nacional e internacional.

Gómez Arévalo (2014) habla sobre la época contemporánea (época actual), la cual ha sido testigo de los innumerables acontecimientos de caos medioambiental, por los desastres ocasionados por el desorden económico, el mal uso de las tecnologías y las malas decisiones políticas en cuanto energía, agua, aire, fauna, flora y tierra.

Por ello es importante tomar la política, la ética, la ecología y la tecnología como soluciones ante esta problemática ambiental. 


\section{ALTERNATIVAS POLÍTICA, ÉTICAS Y ECOLÓGICAS}

Primeramente, es importante definir los conceptos de política, ética y ecología. Según Oyarzún (2018), la ética es la rama de la filosofía que estudia la conducta humana y su moral, encontrando una manera de juzgar la misma, también, determina como deben actuar los miembros pertenecientes a la sociedad, esto debido a que es una ciencia de comportamiento moral.

Por otro lado, Gamboa-Bernal (2011) define el concepto de ecología como la rama de la ciencia que estudia la relación entre los seres vivos y el medio ambiente que los rodea, tomando en cuenta los factores bióticos (los organismos) y los factores abióticos (relieve, clima, entre otros).

La política, por su parte, es una ciencia de gobernación para la toma de decisiones y organización de las sociedades humanas y estudia asuntos que afectan a la sociedad para darles una solución (AriasArbeláez, 2019).

Igualmente, es significativo diferenciar entre la ética ecológica y la ética medioambiental, la ética ecológica es entendida como una ética "del medio ambiente", donde se considera que los animales (no humanos) y la vegetación del planeta también deben poseer valor moral y ser respetados. En cambio, la ética medioambiental es una ética "para el uso del medio ambiente" donde lo único que importa son los intereses del ser humano (Franco da Costa, 2009).

La ecología humana y ecología ambiental son un binomio clave. La ecología humana como su nombre lo dice, de los humanos, es diferente, ya que estos no dependen del medio ambiente para habitarlo, sino que tiende a transformar el medio para hacerlo más adecuado para vivir, lo que interfiere en la balanza ambiental; la ecología ambiental es la ya antes mencionada, donde su objetivo es obtener conocimientos acerca de la realidad del ambiente. El conjunto de estas dos ecologías crea modelos para detener la destrucción de la naturaleza e interactuar con ella de manera sostenible, en beneficio mutuo y de las futuras generaciones (Gamboa-Bernal, 2011).

Una relación socioecológica, según Crane (2010), es la interacción entre factores biofísicos (clima, relieve, suelo y su uso y cobertura vegetal) y sociales, esta relación es de verdadera importancia ya que promueve que sectores menos apreciados por la sociedad, como rurales, pobres, costeros o indígenas, y en general, la humanidad, construya sistemas 
resilientes y adaptativos en respuesta al cambio climático, es decir, sistemas para combatir el cambio climático.

La ecoética hace referencia a las interconexiones del mundo ético respecto a las interacciones del ser humano con la misma naturaleza y con el universo, donde se utiliza el término "casa común para todos" (todos los componentes del planeta), que dicta que todas esas especies están en el mismo nivel de importancia y que el humano debe verlas como sus iguales, no como sus inferiores (Gómez Arévalo, 2014).

La economía política estudia las relaciones sociales de producción, regidas por decisiones colectivas para el aprovechamiento de la naturaleza y el bienestar. La economía política del ambiente retoma la tradición de la economía política, pero hace entender y controlar la desigualdad en la distribución de la riqueza y el poder para entender y controlar la degradación ambiental. Donde la primera alternativa para proteger la naturaleza es la realización de decisiones por mecanismos democráticos para reducir los excesos de las personas ricas y poderosas sobre las personas pobres y/o excluidas, ya que estas últimos son las que reciben la mayor parte de los efectos negativos de la degradación del medio ambiente (Arias-Arbeláez, 2019).

También, es bueno pensar en la ecología histórica, que enfatiza en la necesidad de la colaboración científica entre disciplinas para una investigación académica más relevante y aplicada, particularmente en servicio a la conservación ambiental y justicia social (sostenible), en conjunto con la etnobiología, esta última se describe como el conocimiento en la comprensión del uso y manejo de los recursos naturales, generada por la población indígena (Armstrong y Veteto, 2015).

Alimonda et al. (2011) mencionan que es necesario una ecología política, donde se tendría un modelo parecido al de las y los antepasados, en el cual construían pautas y modos de convivencia con la naturaleza, los cuales eran y son expresados en saberes, tecnologías, formas de organización social y confecciones míticas y simbólicas. Elaboraron sistemas complejos de aprovechamiento de los recursos naturales de manera sustentable, que para esta época se deben transformar a sostenibles.

Algunos ejemplos de países que aplican estas políticas y éticas ecológicas son Ecuador y Chile. En Ecuador su nueva Constitución presenta por primera vez en América Latina una preferencia 
hacia el biocentrismo (todo ser vivo merece respeto moral), donde encuadran los conceptos de los derechos de la naturaleza y el derecho a su restauración, se genera una nueva coyuntura con los saberes tradicionales y ofrece un contexto para las políticas y la gestión ambiental que se basa en la frase "sumak kawsay", quiere decir buena vida, es un término indígena de pueblos ecuatoriales y bolivianos, que ilustra "el buen vivir", un plan de vida socialmente justo y ambientalmente sostenible; y en nuevas estrategias de desarrollo, aceptan el término Pachamama para referirse a la naturaleza y utilizan ambos. Este giro biocéntrico proyecta una alternativa a la moda actual relacionada con las nuevas formas de valoración ambiental en conjunto con los saberes indígenas (Gudynas, 2009).

Chile, por su parte, relaciona la sustentabilidad empresarial, además de tener rentabilidad financiera, ofrece apoyo social a los colectivos locales, como oportunidades de trabajo, y protege y conserva la biodiversidad del país, esto en el caso de una economía internacionalizada como la chilena (Suárez, 2013). 


\section{ALTERNATIVA TECNOLÓGICA}

Por lo anterior, es importante el implemento de una bioeconomía la cual, según Mohammadian (2007), tiene como objetivo ser la conexión entre la ciencia de la biología y la ciencia literaria de la economía, donde se hace un aprovechamiento y uso inteligente de recursos naturales con la utilización de conocimientos y tecnologías para una producción sostenible de bienes y servicios. Esta economía representa un cambio fundamental en la ideología del ser humano en todo lo que se relaciona con las actividades socioeconómicas, biológicas, éticas y sostenibles.

Retomando el concepto de bioeconomía, que promueve la utilización de tecnologías para tener un desarrollo sostenible, una buena opción de tecnología son los Sistemas de Información Geográfica (SIG o GIS en inglés). Según Soto (2019), los SIG son tecnología de manejo de información geográfica formada por equipos electrónicos (hardware) programados adecuadamente (software) que permiten manejar una serie de datos espaciales (información geográfica) y realizar un análisis geográfico de interés con éstos.

Existen diferentes tipos de programas (software) que se pueden utilizar como lo es QGis, ArcGis, gvSig, entre otros. Para crear u obtener los datos espaciales e ingresarlos a estos programas y así generar productos. Se puede ejecutar de diferentes maneras, como ir al área de estudio y tomar puntos con un con un Sistema de Posicionamiento Global (GPS, por sus siglas en inglés), hojas cartográficas, fotografías aéreas, imágenes espectrales, fuentes nacionales o internacionales de datos geográficos, entre otros (Soto, 2019).

Para González, Fernández Freire, Martín-Forero Morente, y Pérez Asensio (2012), los datos pueden visualizarse de tipo vector representados por puntos, líneas y polígonos, o de tipo ráster que son las imágenes satelitales y los modelos de elevación que poseen píxeles con valores asociados.

Aunque la utilización de esta tecnología es relativamente reciente en el ámbito científico, es usualmente utilizada para estudios de problemáticas medioambientales, recursos naturales y ciencias agropecuarias, por ejemplo: ordenamiento territorial, gestión de recursos naturales, agricultura de precisón, fragmentación del bosque, impacto ambiental, trazabilidad bovina y porcina, manejo de recursos forestales, estudios hidrológicos y gestión de cuencas, sistema de emergencia ante el cambio climático, entre otros estudios (Soto, 2019) 


\section{METODOLOGÍA}

El eje principal del presente artículo se basó en el análisis sobre el impacto ético, político y ecológico en la ciencia y tecnología contemporánea. Donde, primeramente, se realizó una búsqueda de artículos y trabajos académicos a través de diferentes fuentes bibliográficas para enriquecer de manera cualitativa la literatura. El trabajo se encaminó en la búsqueda de información para explicar la lamentable situación del medio ambiente, los conceptos filosóficos, tales como: política, ética, ecología y sus derivadas; asimismo, para definir el sistema económico basado en la bioeconomía y lo que son los Sistemas de Información Geográfica.

Mediante un estudio de tres casos procedentes de artículos y trabajos académicos, se logró definir a profundidad la utilidad de los SIG, estos caos se eligieron bajo el criterio único de interés académico. El primer caso realizado por Carvajal Venegas (2017) donde se analizó la pérdida de suelos de la subcuenca del río Tempisquito en Guanacaste, Costa Rica; el segundo caso estudiado por está relacionado a la producción de agua de una cuenca hidrológica y, por último, un tercer caso, el cual elaboró su estudio de suelos en una modelación creada en un laboratorio con el fin de determinar prácticas para la conservación del suelo. 


\section{DISCUSIÓN Y RESULTADOS}

\section{SISTEMAS DE INFORMACIÓN GEOGRÁFICA Y CONSTRUCCIÓN DE ALTERNATIVAS A PROBLEMÁTICAS}

Caso 1: Grado de erosión en la subcuenca del río Tempisquito, Guanacaste, Costa Rica.

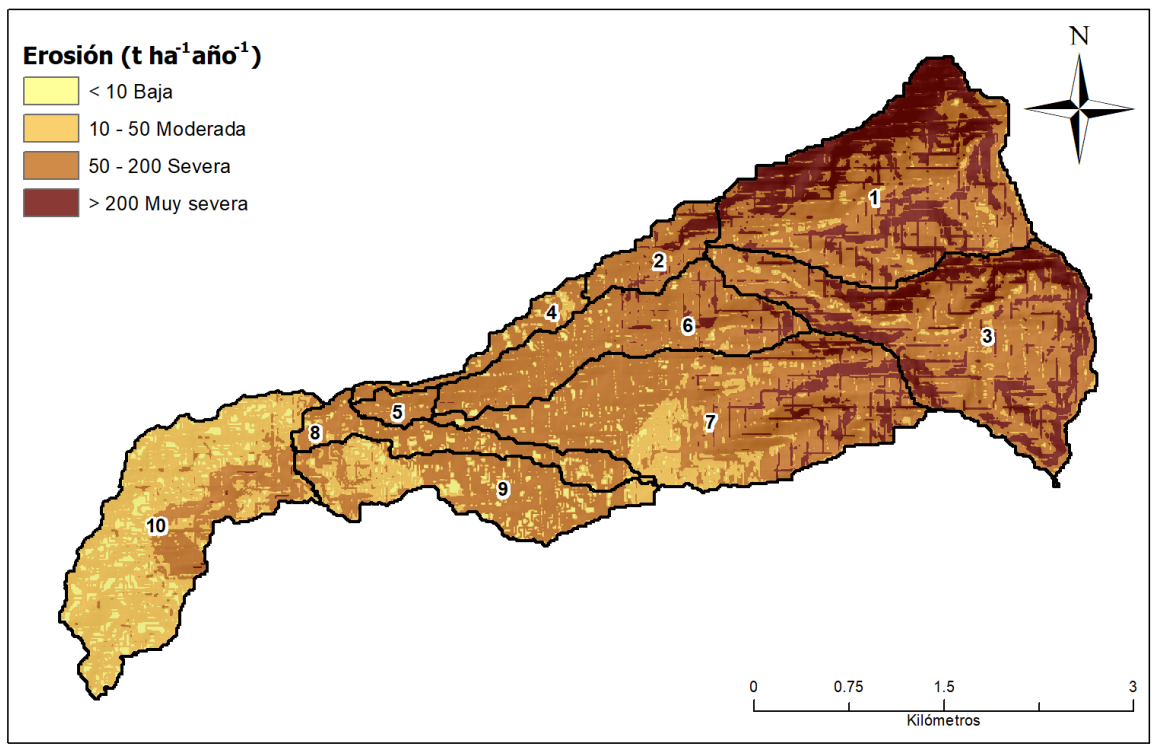

Figura 1. Pérdidas de suelo por erosión en la subcuenca del río Tempisquito, Guanacaste, Costa Rica. Fuente: Carvajal Venegas, 2017.

En este caso realizado en la tesis de Carvajal Venegas (2017) se utilizó el programa de Sistemas de Información Geográfica QGIS 2.6.1, una herramienta de calidad y, sobre todo, gratuita; junto a la extensión QSWAT 1.4, una versión del modelo SWAT (Soil and Water Asessment Tool) el cual simula el ciclo hidrológico basándose en la ecuación de balance de agua. Estas herramientas se utilizaron para la creación de distintos mapas, en este caso, para hacer un estudio sobre la pérdida de suelo por erosión en la subcuenca del río Tempisquito en Guanacaste, Costa Rica. En este se puede observar la forma de la subcuenca la cual se encuentra dividida por sus microcuencas, su dimensión con una escala en kilómetros y, a su vez, muestra sus categorías de erosión, las cuales están bien especificadas en la leyenda que va desde baja $(<10)$ a muy severa (> 200). Como resultado se obtiene que la erosión media de la subcuenca es de 122,2 t ha-1 año-1 (toneladas por hectárea por año), 


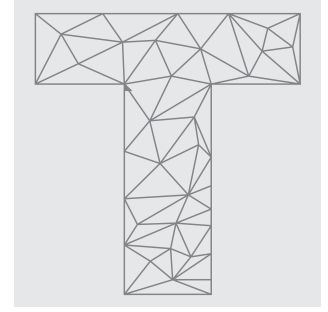

siendo clasificada como una erosión severa. Igualmente, se determina que la microcuenca que reporta más pérdida de suelo es la 1 con $258 \mathrm{t}$ ha-1 año-1 y la que aporta menos erosión es la 10 con 38 t ha-1 año-1.

Este estudio permite que la interpretación de la erosión de un determinado lugar sea más fácil de analizar, muestra los lugares específicos con mayor y menor problema de pérdida del suelo si el sitio presenta o no erosión significativa; asimismo, junto con otros estudios (pendiente, cobertura, uso del suelo, entre otros) se podría observar la relación existente con la erosión y, así, determinar la causa de la pérdida del suelo y proponer planes de manejo para su conservación.

Además, desde un punto de vista de la bioeconomía, antes de ejecutar cualquier actividad productiva, la implementación del estudio de la erosión, antes de ejecutar cualquier actividad productiva, el estudio de erosión permitirá conocer si el sitio presenta una erosión severa, las zonas con mayores problemas de erosión para trabajar en su conservación y para evitar que se sobreexplote un suelo que presenta ya un problema. Con esto se puede establecer cuáles actividades son las ideales para determinado sitio $y$, no solo evitar el desgaste del suelo, sino también posibles accidentes, poniendo en balance los intereses económicos con la protección ambiental de los suelos. 
Caso 2: Estudio hidrológico de la subcuenca del Sector Prusia, Parque Nacional Volcán Irazú, Cartago, Costa Rica.
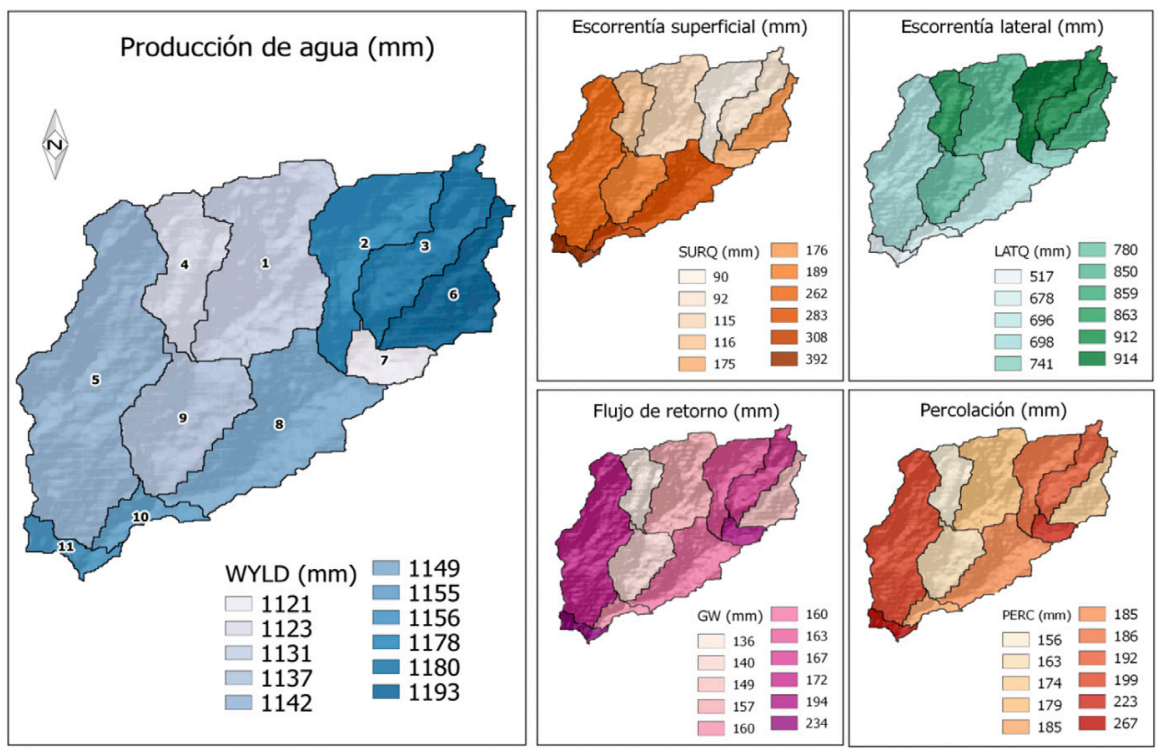

Figura 2. Producción de agua media anual y sus componentes en la subcuenca del Sector Prusia, Parque Nacional Volcán Irazú. Fuente: Valverde Gamboa, 2018.

En la Figura 2 se puede observar un estudio realizado en la tesis de Valverde Gamboa (2018) sobre la producción de agua media anual y sus componentes en la subcuenca del Sector Prusia, Parque Nacional Volcán Irazú, Cartago, Costa Rica en el cual, igualmente, se utilizó el programa de QGIS 2.6.1. y el modelo SWAT.

En este estudio se determina la producción de agua de la subcuenca y cuáles microcuencas son las de mayor producción de agua $(2,3$ y 6$)$ y lo relaciona con otro estudio que indica que esas zonas están cubiertas por vegetación arbórea que disminuye el escurrimiento superficial del agua y la erosión del suelo; así como las microcuencas con menos producción (como la 10 y 11), que están relacionadas con su cobertura que son pastos y cultivos los cuales aumenta la escorrentía superficial y disminuyen la capacidad de retención de agua del suelo.

También, muestra otros componentes como la escorrentía superficial, flujo lateral, flujo de retorno y percolación, los cuales dan un 
mejor análisis del porqué de la producción de agua de subcuenca del río Tempisquito y están asociados al tipo de suelo, la pendiente y la cobertura.

Este mapeo, entonces, permite conocer cuánto se produce de agua en una zona en específico y junto con otros componentes y estudios (suelo, pendiente, cobertura) se explica por qué determinado sitio produce menos o más agua. Con esta información se puede idear y establecer un plan de manejo para la subcuenca o microcuencas para la protección de este recurso natural tan importante.

Caso 3: Respuesta hidrológica del suelo de una cuenca modelada en laboratorio con diferentes tratamientos de sustratos.
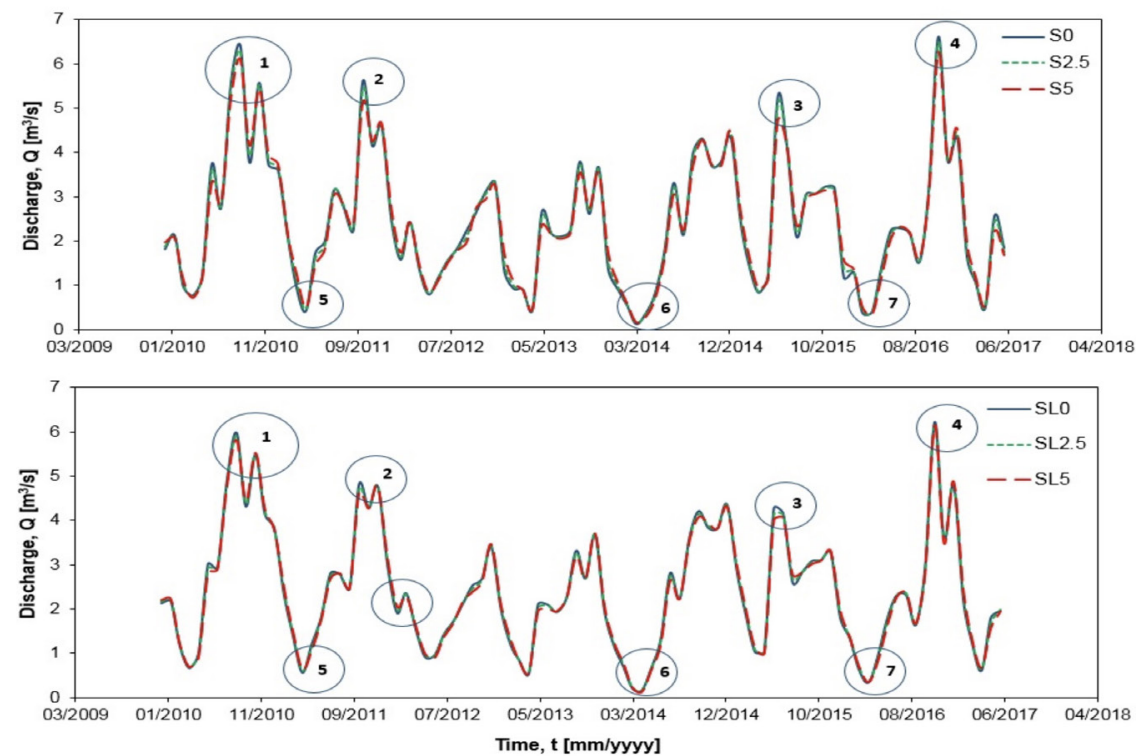

Figura 3. Descarga de agua simulada (Q) aplicando seis escenarios (SO, S2.5, S5, SL10, SL2,5 y SL5) para el terreno ocupado por cultivos y pastizales. Gráfico superior: descarga de agua de los tratamientos arenosos. Gráfico inferior: descarga de agua de los tratamientos franco arenosos. Fuente: Villagra-Mendoza, Carvajal-Venegas, Beck-Broichsitter y Horn, 2020. 
En el trabajo realizado por Villagra-Mendoza, Carvajal-Venegas, Beck-Broichsitter y Horn (2020) se estudió durante 7 años el efecto de sustratos en diferentes cantidades de biocarbón (astillas de madera de mango) sobre la infiltración del agua en un suelo arenoso y en un suelo franco arenoso de una cuenca hidrográfica hipotética creada en un laboratorio. La infiltración se calculó después de simular ciclos de época lluviosa y de época seca. Igualmente, los parámetros hidráulicos del suelo modificados con biocarbonos, obtenidos en el laboratorio, se utilizaron para simular la descarga de agua de la cuenca hipotética con el uso del SWAT.

En la figura anterior se observa que los eventos de noviembre a abril (estación seca) provocaron picos de escorrentía significativamente más pequeños que los eventos de mayo a octubre (estación lluviosa). En la Figura 3, el gráfico superior muestra la serie de tiempo de la descarga de agua $(\mathrm{Q})$ para los tratamientos arenosos $(\mathrm{S})$ y el gráfico inferior de la Figura 3 el período correspondiente a los tratamientos franco arenosos (SL).

En este estudio se determina que los picos altos suceden durante la época lluviosa y los flujos bajos ocurren durante la época seca. Además, para los tratamientos arenosos (gráfico superior de la Figura 3) y los franco arenosos (gráfico inferior de la Figura 3) la cuenca mantuvo la misma forma de descarga de agua (Q), pero su magnitud varió, obteniendo una descarga mayor en las mezclas arenosas (S), principalmente durante la temporada de lluvias.

Se escogieron cuatro picos altos durante la temporada de lluvias $(1,2,3$ y 4$)$ y tres salidas bajas durante la temporada seca $(5,6$ y 7$)$, estos picos altos y bajos encerrados en un círculo se utilizan como ejemplo para un análisis más detallado con otros gráficos en el trabajo, donde se determina que los suelos sin enmiendas (S0 y SL0) presentaron los picos más altos de descarga con respecto a los suelos con enmiendas (S2.5, S5, SL2.5 y SL5). Además, se demuestra que los sustratos franco arenosos (SL) pudieron retener más agua en el suelo y transportar menos agua en el suelo que los sustratos arenosos (S).

Del análisis de gráficos se determina que en condiciones de suelo seco las enmiendas de biocarbón pueden transportar más agua que los suelos sin tratamientos, debido a su mayor conductividad hidráulica. Asimismo, la enmienda de biocarbón como una alternativa para el manejo de la conservación del suelo puede ayudar a mejorar la retención de agua del suelo en condiciones de sequía prolongada. 
Por lo tanto, este estudio señala que el SWAT es un programa con grandes potencialidades para analizar los tratamientos con sustratos al suelo y determinar los mejores tratamientos para la conservación del suelo, dando a la población opciones para conservar y cuidar el suelo, en especial en época seca.

Finalmente, con estos casos se demuestra que los Sistemas Información Geográfica son una buena herramienta para estudiar el medio ambiente, ya que los estudios ambientales pueden ser georreferenciados utilizando un sistema de coordenadas espaciotemporal. Además, los SIG mejoran las investigaciones científicas, porque permiten el desarrollo de otras oportunidades de conocimiento o perspectivas, aplicando otros puntos de análisis y proporcionando nuevos datos que sin esta tecnología muy posiblemente permanecería invisibles, en otras palabras, facilitando la interpretación de la realidad (González, Fernández Freire, MartínForero Morente Pérez Asensio, 2012). 


\section{CONCLUSIONES}

Debido al sistema económico mundial actual y la falta de conciencia ambiental de la población, el planeta presenta una crisis ambiental que atenta contra la vida de orden global, por ende, es importante tomar una ética y una política ecológica, donde todos los seres vivos sean debidamente respetados y se logre tener un desarrollo económico socialmente justo y sostenible con el ambiente.

La bioeconomía al ser un sistema económico que usa y aprovecha de forma inteligente los recursos naturales y utiliza las tecnologías para obtener una producción sostenible es una gran alternativa para resolver problemas medioambientales.

Además, en el análisis de los casos se observa que los SIG son eficientes para realizar estudios sobre el grado de erosión de un sitio (Caso 1), estudios hidrológicos acerca de la producción de agua media anual de una cuenca (Caso 2) y analizar la respuesta hidrológica del suelo con diferentes tratamientos de sustratos para su conservación (Caso 3). Por lo tanto, se demuestra que los Sistemas de Información Geográfica son una excelente herramienta para la elaboración de estudios ambientales. 


\section{REFERENCIAS BIBLIOGRÁFICAS}

Alimonda, H. (2011). La naturaleza colonizada Ecología política y minería en América Latina. Buenos Aires. CLACSO. Recuperado de http://biblioteca.clacso.edu.ar/ar/libros/grupos/alimonda.pdf

Arias-Arbeláez, F. A. (2019). Economía política del ambiente: voz y eco en el análisis social de los problemas ambientales. Hallazgos, (16), 27-42. Recuperadodehttps://search.proquest.com/docview/2239547284

Carvajal Venegas, D. (2017). Dinámica hídrica bajo condiciones climáticas cambiantes en la subcuenca del río Tempisquito, cuenca del Tempisque, Guanacaste, Costa Rica (Tesis de maestría). Centro Agronómico Tropical de Investigación y Enseñanza, Turrialba, Costa Rica. Recuperado de http://repositorio.bibliotecaorton.catie.ac.cr/bitstream/ handle/11554/8705/Dinamica_hidrica_bajo_condiciones_climaticas.pdf? sequence $=1$ \&isAllowed $=y$

Crane, T. A. (2010). Of Models and Meanings: Cultural Resilience in Social-Ecological. Ecology and Society, 15(4), 19-35. Recuperado de https://www.jstor.org/stable/26268225?seq=1\#metadata_info_ tab_contents

Cuervo Bernal, M. y Gutiérrez Rodríguez, A. (2016). La ecología como práctica educativa interdisciplinar entre educación física y tecnología. Praxis Pedagógica, 16(18), 91-99. Recuperado de https://www.researchgate.net/publication/321014734_La_ecologia_como_practica_educativa_interdisciplinar_entre_educacion_fisica_y_tecnologia

Franco da Costa, C. A. (2009). ¿Ética ecológica o medioambiental? Acta Amazonica, 39(1), 113-120. Recuperado de https://www.scielo. br/scielo.php?pid=S0044-59672009000100012\&script=sci_abstract\&tlng=es 
Gamboa-Bernal, G. A. (2011). Ecología humana y ecología ambiental: binomio clave. Persona y bioética, 15(1), 5-9. Recuperado de http:// www.scielo.org.co/pdf/pebi/v15n1/v15n1a01.pdf

Gómez Arévalo, J. A. (2014). Reflexiones en torno a la ecoética y sus aportes en la época contemporánea. Revista Latinoamericana de Bioética, 14(2), 66-79. Recuperado de https://www.redalyc.org/ pdf/1270/127032031006.pdf

González, I.; Fernández Freire, C.; Martín-Forero Morente, L. y Pérez Asensio, E. (2012). Los Sistemas de Información Geográfica y la Investigación en Ciencias Humanas y Sociales. Recuperado de http://digital.csic.es/bitstream/10261/64940/1/Los\%20SIG\%20y\%20la\%20 Investigacion\%20en\%20Ciencias\%20Humanas\%20y\%20Sociales. pdf

Gudynas, E. (2009). La ecología política del giro biocéntrico. Revista de Estudios Sociales, (32), 34-47. Recuperado de https://revistas.uniandes.edu.co/doi/pdf/10.7440/res32.2009.02

Mohammadian, M. (2005). La Bioeconomía un nuevo paradigma socioeconómico para el siglo XXI. Encuentros multidisciplinares, 7(19), 5770. Recuperado de http://www.encuentros-multidisciplinares.org/ Revistan\%BA19/Mansour\%20Mohammadian.pdf

Oyarzún, P. (2018). Ecología y ética. Magallania, 46(1), 279-287. Recuperado de http://www.magallania.cl/index.php/magallania/article/ view/1040

Soto, C. (2019). Curso de Sistemas de Información Geográfica. [Diapositivas de PowerPoint]. Repositorio Escuela de Ingeniería Forestal, tecDigital. Recuperado de https://drive.google.com/file/d/11HskxGRrelhsWwnYqkIWkzsJljtHubFj/view?usp=sharing 


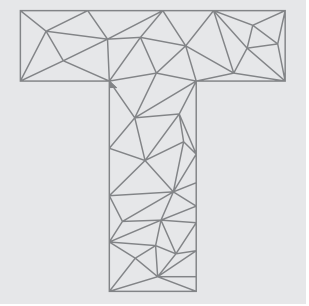

Suárez, A. (2013). Sustentabilidad empresarial, seguridad energética y ética ambiental en Chile. Acta bioethica, 19(2), 199-208. Recuperado de https://scielo.conicyt.cl/scielo.php?script=sci_arttext\&pid=S1726-569X2013000200004

Valverde Gamboa, E. D. (2018). Modelación hidrológica para cuantificar la producción de agua y sedimento actual y futuro a partir de cambios de cobertura forestal, sector Prusia, Parque Nacional Volcán Irazú (Tesis de pregrado). Instituto Tecnológico de Costa Rica, Cartago, Costa Rica. Recuperado de https://drive.google.com/file/d/10Vkwz-HI9GuOJI0_QuM9F_IlzrT8KnpL/view?usp=sharing

Villagra-Mendoza, K.; Carvajal-Venegas, D.; Beck-Broichsitter, S. y Horn, R. (2020). A simulated effect of wetting and drying periods for two textural soils amended with biochar at a catchment scale. CIGR Journal, 22(1), 9-21. Recuperado de https://cigrjournal.org/index.php/ Ejounral/article/view/5562 